\title{
Overactivation of poly(adenosine phosphate-ribose) polymerase 1 and molecular events in neuronal injury after deep hypothermic circulatory arrest: Study in a rabbit model
}

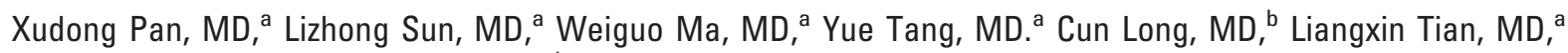
Ningning Liu, MD, ${ }^{a}$ Zhengyi Feng, MD, and Jun Zheng, $\mathrm{MD}^{\mathrm{a}}$

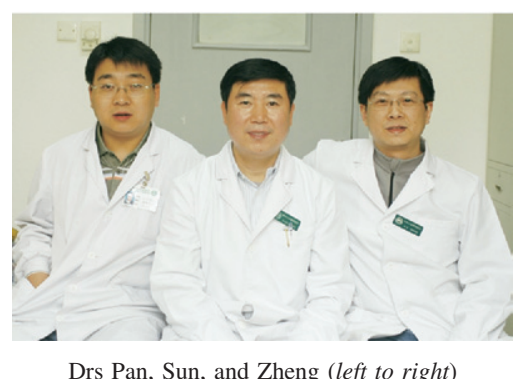

Drs Pan, Sun, and Zheng (left to right)

Supplemental material is available online.
From the Department of Cardiovascular Surgery and Aortic Surgery Center ${ }^{\mathrm{a}}$ and the Department of Cardiopulmonary Bypass, ${ }^{b}$ Cardiovascular Institute and $\mathrm{Fu}$ Wai Hospital, Chinese Academy of Medical Sciences and Peking Union Medical College, Peking, China.

Supported by grants from the Natural Science Foundation of China (30070751) and the Specialized Research Fund for the Doctoral Program of Higher Education (20040023017) of China.

Received for publication Feb 7, 2007; revisions received April 18, 2007; accepted for publication May 9, 2007.

Address for reprints: Jun Zheng, MD, Department of Cardiovascular Surgery and Aortic Surgery Center, Cardiovascular Institute and Fu Wai Hospital, 167 Beilishi Road, Peking, China 100037. (E-mail: dr.zhengjun@gmail.com)

J Thorac Cardiovasc Surg 2007;134:1227-33 $0022-5223 / \$ 32.00$

Copyright @ 2007 by The American Association for Thoracic Surgery

doi:10.1016/j.jtcvs.2007.05.062
Objective: Although deep hypothermic circulatory arrest has been known to induce neuronal injury, the molecular mechanism of this damage has not been identified. We studied the key molecular mediators through cellular energy failure, excitotoxicity, and overactivation of poly(adenosine diphosphate-ribose) polymerase 1 in brain tissues of a rabbit model of deep hypothermic circulatory arrest similar to clinical settings.

Methods: We established 2 models of cardiopulmonary bypass $(\mathrm{n}=15)$ and deep hypothermic circulatory arrest $(\mathrm{n}=15)$ associated with cerebral microdialysis in rabbits. Deep hypothermic circulatory arrest lasted for 60 minutes. The measurements of glucose, lactate, pyruvate, and glutamate collected by means of microdialysis were quantified by using a microdialysis analyzer and high-performance liquid chromatography. The overactivation of poly(adenosine diphosphate-ribose) polymerase 1 was assessed by detecting immunostaining of poly(adenosine diphosphate-ribose). Histologic studies were used to identify neuronal morphologic changes and terminal deoxynucleotidyl transferase-mediated deoxyuridine triphosphate nick end-labeling staining and poly(adenosine diphosphate-ribose) polymerase 1 Western blotting were used to identify apoptotic cells and early apoptotic signals.

Results: Deep hypothermic circulatory arrest significantly increased the lactate/ pyruvate and lactate/glucose ratios and the glutamate value, whereas cardiopulmonary bypass did not $(P<.05)$. Deep hypothermic circulatory arrest significantly increased the numbers of poly(adenosine diphosphate-ribose)-positive and apoptotic neurons compared with cardiopulmonary bypass $(P<.05)$. The cleavage of poly(adenosine diphosphate-ribose) polymerase 1 was only found in the deep hypothermic circulatory arrest group. More injured neurons were found in the deep hypothermic circulatory arrest group (histologic scores, $P<.05$ ).

Conclusions: This study demonstrated that deep hypothermic circulatory arrest results in an overactivation of poly(adenosine diphosphate-ribose) polymerase 1, and that there were molecular events consisting of cellular energy failure, excitotoxicity, overactivation of poly(adenosine diphosphate-ribose) polymerase 1 , and necrosis and/or apoptosis in neuronal injury.

$\mathrm{D}$ eep hypothermic circulatory arrest (DHCA) has been an important strategy in surgical procedures for complex congenital cardiac malformations and aortic arch diseases since its introduction in the 1940s. Although DHCA afforded a neuroprotective effect and enabled the surgeon to obtain a bloodless operative field that allowed for precise anatomic manipulation, it was always 


\author{
Abbreviations and Acronyms \\ ADP = adenosine diphosphate \\ ATP = adenosine triphosphate \\ $\mathrm{CPB}=$ cardiopulmonary bypass \\ DHCA = deep hypothermic circulatory arrest \\ $\mathrm{NAD}^{+}=$nicotinamide adenine dinucleotide \\ PAR = Poly(ADP-ribose) \\ PARP-1 = poly(adenosine diphosphate-ribose $)$ \\ polymerase 1 \\ PBS = phosphate-buffered saline \\ SEM $=$ standard error of the mean \\ TUNEL $=$ terminal deoxynucleotidyl \\ transferase-mediated deoxyuridine \\ triphosphate nick end-labeling
}

associated with immediate and late neuronal complications. ${ }^{1-4}$ Preventing cerebral injury remains difficult because the underlying mechanisms have not been fully elucidated.

Neuronal injury after DHCA is generally ascribed to cerebral hypoxia, ischemia, and oxidative stress after ischemia-reperfusion, except for cardiopulmonary bypass (CPB) perturbation, anesthesia management, and preoperative neurologic deficits. ${ }^{5,6}$ Ischemia and ischemia-reperfusion injury initiate a cascade of cellular and molecular events, of which excitotoxicity caused by overstimulation of excitory amino acids, mainly glutamate, has been a wellknown fundamental common process in all kinds of ischemic brain injury. ${ }^{4,6,7}$ Of note, the role of excitotoxicity in neuronal injury after DHCA was demonstrated by Tseng and colleagues. ${ }^{8}$ Apoptosis and necrosis, the possible sequelae of excitotoxicity, ${ }^{9}$ were also identified to play a crucial role in neuronal injury after DHCA. ${ }^{10,11}$ Ischemia, cellular energy failure, excitotoxicity, necrosis, and apoptosis might constitute a path to neuronal injury after DHCA.

Excitotoxicity was seen as a final pathway in most neuronal injuries in the 1990s, ${ }^{12}$ but in recent studies of normothermic ischemic brain injury, a key event of overactivation of poly(adenosine diphosphate [ADP]-ribose) polymerase 1 (PARP-1) has been gradually identified as a link between excitotoxicity and cell death, which emerges as central in cell death that occurs in the nervous system. ${ }^{13,14}$ Activated PARP- 1 cleaves nicotinamide adenine dinucleotide $\left(\mathrm{NAD}^{+}\right)$, which contributes to DNA repair and to maintenance of genomic stability. On the other hand, overactivation of PARP-1 results in rapid depletion of intracellular $\mathrm{NAD}^{+}$and consequently of adenosine triphosphate (ATP) and finally to cell dysfunction or death. ${ }^{13,15}$ This cellular "suicide" mechanism has been implicated in the pathomechanism of cerebral ischemia, stroke, traumatic nervous system injury, and so on, ${ }^{13-16}$ but whether the overactivation of PARP-1 plays a role in neuronal injury after DHCA is still unclear.
Although molecular events, brain cellular energy disorders, and excitotoxicity have been proved to induce neuronal injury after DHCA by different groups under different conditions, it is unknown whether these molecular events concurrently play a role in this scenario. In this study we investigated the possibility that there was an overactivation of PARP-1 and molecular events in neuronal injury after DHCA, which were simultaneously composed of cellular energy failure, excitotoxicity, overactivation of PARP-1, and necrosis and/or apoptosis occurring in the same condition.

\section{Materials and Methods}

Male New Zealand white male rabbits $(\mathrm{n}=30)$ weighing 3.0 to $3.5 \mathrm{~kg}$ were used. All animal procedures were approved by the Animal Care and Use Committee of Peking Union Medical College. All animals received humane care in compliance with the "Guide for the care and use of laboratory animals" published by the National Academy of Science (National Institutes of Health publication no. 85-23, revised 1985).

\section{Cerebral Microdialysis}

Animals were deeply anesthetized with pentobarbital $(30 \mathrm{mg} / \mathrm{kg}$ administered intravenously) and placed in a stereotaxic apparatus (Medical Equipment Research Institute). After positioning the dorsal hippocampus (CA1) according to the Sawyer stereotaxic atlas of the rabbit, ${ }^{17}$ a guide cannula was implanted (CMA/12 Guide Cannula, CMA Microdialysis) $4 \mathrm{~mm}$ posterior from the bregma, $4 \mathrm{~mm}$ right lateral from the middle sagittal plane, and 6 $\mathrm{mm}$ ventral from the bregma bone surface. The guide cannula was fixed with dental cement and assembled in a self-made protecting jacket. After the procedure, the rabbits were housed in individual cages with free access to food and water. At 36 hours after implantation of the guide cannula, the core of the guide cannula was replaced with a probe (CMA/12 microdialysis probe, 4-mm membrane length; CMA Microdialysis) and then perfused with artificial cerebrospinal fluid (containing $147 \mathrm{mmol} \mathrm{NaCl}, 2.7 \mathrm{mmol}$ $\mathrm{KCl}, 0.85 \mathrm{mmol} \mathrm{MgCl}_{2}, 1.2 \mathrm{mmol} \mathrm{CaCl}_{2}$, and $0.6 \mathrm{mmol}$ ascorbic acid [ $\mathrm{pH}$ 7.4]) at a rate of $2 \mu \mathrm{m} / \mathrm{min}$ by using a microperfusion pump (CMA/102, CMA Microdialysis). The collection of basal samples was initiated 90 minutes after microdialysis probe positioning and pumping. Microdialysis was used from consciousness to recovery from CPB. Samples of dialysate were collected every 30 minutes and stored at $-70^{\circ} \mathrm{C}$.

\section{CPB and DHCA}

Each rabbit was given diazepam $(2 \mathrm{mg} / \mathrm{kg}$ ) intramuscularly as premedication and anesthetized with intravenous pentobarbital (25 $\mathrm{mg} / \mathrm{kg})$, fentanyl $(20 \mu \mathrm{g} / \mathrm{kg})$, and pipercuronium bromide $(0.15$ $\mathrm{mg} / \mathrm{kg}$ ). Continuous anesthesia was maintained with diazepam and fentanyl. After endotracheal intubation with an intratracheal tube (4-mm internal diameter), a neonatal mechanical ventilator was used (Inter3, Bird). Tidal volume was set at 10 to $15 \mathrm{~mL} / \mathrm{kg}$, and respiratory frequency was set at 30 to 45 breaths/min with a $50 \%$ oxygen concentration. Ventilation was adjusted to keep the $\mathrm{PaCO}_{2}$ and $\mathrm{pH}$ within the physiologic range. We carried out a stepwise management of arterial blood gases using the alpha-stat strategy 
when body temperature was greater than $28^{\circ} \mathrm{C}$ and the pH-stat strategy when body temperature was less than $28^{\circ} \mathrm{C}$. Arterial blood gases and hematocrit values were monitored intermittently. A 20-gauge cannula was inserted in the right or left femoral artery and connected to a fluid-filled pressure transducer to monitor arterial blood pressure and take blood samples; electrocardiographic results and nasopharynx and rectal temperatures were monitored (CSI8100, Criticare). Temperature was maintained at $37.5^{\circ} \mathrm{C}$ to $38.5^{\circ} \mathrm{C}$ before $\mathrm{CPB}$ with an electrical heating exchanger. Limb lead II electrocardiographic data were continuously monitored by means of subcutaneous needle electrodes. Ringer's solution was used as maintenance fluid through the ear vein. The cardiopulmonary circuit consisted of a double small roller pump (Shiley; Stockert, Munich, Germany), a blood reservoir made of a 50-mL syringe, a small heat exchanger connected with a thermostatic water pump (type 20-600, Jostra), and a membrane oxygenator for small animals. All connected silicone catheters were 3 $\mathrm{mm}$ in internal diameter to reduce the priming volume (the heat exchanger, membrane oxygenator, and catheters were provided by Xijing Medical Appliance Corp). All catheters were flushed with $10 \mathrm{U} / \mathrm{mL}$ heparinized saline to prevent clotting during the experiment. The priming volume of the $\mathrm{L}$ group was $75 \mathrm{~mL}$ without blood and consisted of $50 \mathrm{~mL}$ of sodium chloride and gelatin and $10 \mathrm{~mL}$ of Ringer's solution, $1.5 \mathrm{~g}$ of mannitol, $0.25 \mathrm{~g}$ of sodium bicarbonate, $0.5 \mathrm{~g}$ of cefazolin, $0.2 \mathrm{~g}$ of magnesium sulfate, $2 \mathrm{mg}$ of furosemide, and $200 \mathrm{U}$ of heparin. The calculated aim for the final hematocrit value of the blood volume was 0.2 to 0.25 . After median sternotomy, heparin was injected intravenously before cannulation (400-600 U/kg). A Prolene (Ethicon) cardiovascular suture (6-0) was used to form 2 purse-string layers in the ascending aorta. An incision suitable for arterial cannulation on the aortic adventitia was made within the purse-string suture. Without the aid of a side clamp, a 14-gauge trochar (2.1 mm outer diameter) used as an aortic cannula was inserted directly, the probe core was drawn in half, and 2 purse-string tourniquets were secured one by one. A plug of venous cannula (DLP16F, Medtronic) was inserted in the right atrium through the auricular appendage. After an activated clotting time of longer than 480 seconds was achieved, CPB was initiated at a flow rate of 80 to $90 \mathrm{~mL} \cdot \mathrm{kg}^{-1} \cdot \mathrm{min}^{-1}$, which was enough to maintain a mean blood pressure of greater than $60 \mathrm{~mm} \mathrm{Hg}$ most of the time. During the cooling period, the flow rate was gradually decreased. After establishment of CPB, a continuous positive airway pressure mode $\left(5 \mathrm{~cm} \mathrm{H}_{2} \mathrm{O}\right)$ with a fraction of inspired oxygen of $21 \%$ was applied to avoid atelectasis, but during DHCA, the pulmonary ventilation was stopped. Pleural and mediastinal blood was drawn off and reinfused into the venous line. In about 30 minutes, rectal temperature was down to $16^{\circ} \mathrm{C}$ to $18^{\circ} \mathrm{C}$ by using a heat exchanger and topical cooling with ice bags and a cooling blanket. DHCA confirmed by means of asystole was maintained for 60 minutes at $16^{\circ} \mathrm{C}$ to $18^{\circ} \mathrm{C}$. $\mathrm{CPB}$ restarted when rewarming increased the temperature to $35^{\circ} \mathrm{C}$ in 30 minutes and then maintained it for 30 minutes on CPB. During rewarming, the mean arterial pressure was kept at more than 50 $\mathrm{mm} \mathrm{Hg}$ with dopamine until rectal temperatures of $35^{\circ} \mathrm{C}$ or greater and blood flows of $60 \mathrm{~mL} \cdot \mathrm{min}^{-1} \cdot \mathrm{kg}^{-1}$ or greater were achieved. Dopamine was administered at a dosage of 5 to $10 \mu \mathrm{g}$ $\min ^{-1} \cdot \mathrm{kg}^{-1}$ when inotropic support was required during the 2 hours in which the animals were off CPB. After 2 hours of weaning from $\mathrm{CPB}$, the animal was killed.

\section{Study Protocol}

Rabbits were assigned to one of 2 groups at random: the DHCA group $(\mathrm{n}=15,10$ for histopathologic study and 5 for Western blotting) and the CPB group ( $\mathrm{n}=15,10$ for histopathologic study and 5 for Western blotting). The procedures of the CPB group were the same as those of the DHCA group, except there was no DHCA. The whole procedure of the DHCA group was as follows:

1. consciousness microdialysis in 90 minutes (base), gathering 3 samples from microdialysis;

2. ready for $\mathrm{CPB}$ and anesthesia in 60 minutes (0-60 minutes), gathering 2 samples;

3. $\mathrm{CPB}$ and cooling in 30 minutes (60-90 minutes), gathering 1 sample;

4. DHCA in 60 minutes (90-150 minutes), gathering 2 samples;

5. CPB and rewarming in 30 minutes (150-180 minutes), gathering 1 sample;

6. assisted circulation for 30 minutes (180-210 minutes), gathering 1 sample; and

7. recovery in 120 minutes (210-330 minutes), gathering 4 samples.

Glutamate from microdialysis was determined by using highperformance liquid chromatography (Alliance HPLC Systems, Waters Corp). Glucose, lactate, and pyruvate from mcrodialysis were measured with a microdialysis analyzer (CMA 600, CMA Microdialysis). The relative recovery of the microdialysis probe was determined in vitro. The values for the level of samples were corrected for the relative recovery and presented.

At the end of the experiment, correct probe placement was checked by means of visual inspection of the probe tracks on 2-mm coronal sections from the hippocampus domain (CA1) of each rabbit. Only rabbits with correct probe placement were considered in the results.

\section{Histologic Examination}

After 2 hours of weaning from CPB, rabbits underwent transcarotid perfusion with $500 \mathrm{~mL}$ of heparinized saline followed by $1000 \mathrm{~mL}$ of cold phosphate-buffered saline (PBS; $0.1 \mathrm{~mol} / \mathrm{L}, \mathrm{pH}$ 7.4) containing $4 \%$ paraformaldehyde. The brains were removed, cut into 2-mm coronal sections, and kept for 2 days in the same fixative solution at $4^{\circ} \mathrm{C}$ before paraffin embedding. After paraffin embedding, paraffin sections (10 $\mu \mathrm{m}$ thick) were cut on the microtome at the level of the midhippocampus (CA1) and stained with hematoxylin and eosin. All slides were scored by 2 observers blinded to the experimental groups. Two brain regions of each hemisphere were chosen for evaluation of neuronal cell damage: the parietal cortex and the hippocampus (CA1). The injured neurons were counted with an image-analysis system (Alpha Innotech). Five serial high-magnification $(400 \times)$ fields per region were examined by use of the following scores: 0 , fewer than 5 damaged neurons; 1,5 to 10 damaged neurons; 2, 10 to 20 damaged neurons; and 3, more than 20 damaged neurons. The score values for special region were summed to a total histologic score for each animal, with a maximum score of 60 (5 high-magnification fields $\times 3$ as worst score $\times 2$ brain regions $\times 2$ hemispheres), which 
allowed for semiquantitative comparison between groups. A normal range of 0 to 12 was taken from 5 healthy untreated rabbits.

\section{Poly(ADP-ribose) Immunohistochemistry}

Poly(ADP-ribose) (PAR), the product of PARP, was detected to assess the activation of PARP. ${ }^{18}$ The paraffin-embedded specimens of parietal cortex and hippocampus biopsy specimens were made in the same way. After sectioning (10 $\mu \mathrm{m}$ thick) of the tissues, slides were deparaffinized, antigen was retrieved by means of incubation in boiling $0.1 \mathrm{~mol} / \mathrm{L}$ sodium citrate $(\mathrm{pH}$ 6), and slides were rinsed in water. Slides were incubated in $10 \%(\mathrm{wt} / \mathrm{vol})$ trichloroacetic acid for 10 minutes to prevent catabolism of the polymer by PAR glycohydrolase. Slides were rinsed in PBS and then incubated for 15 minutes in $1.5 \%$ ( $\mathrm{vol} / \mathrm{vol}$ ) hydrogen peroxide in methanol for quenching endogenous peroxidase activity. Nonspecific binding sites were blocked with $2 \%$ (vol/vol) normal goat serum in PBS for 1.5 hours at $37^{\circ} \mathrm{C}$. The slides were incubated overnight at $4^{\circ} \mathrm{C}$ with mouse antibody against PAR (dilution 1:250, Clone 10H; Tulip BioLabs, Inc). Then the slides were washed in PBS and biotinylated sheep anti-mouse secondary antibody (Amersham, RPN1001), diluted 1:200 in PBS, and applied to the tissues for 2 hours. The tissues were then incubated for 30 minutes with avidin-horseradish peroxidase conjugate (ABC kit, Vector Laboratories), and the reaction was visualized with 3-amino-9-ethyl-carbazole. Slides were counterstained with Harris hematoxylin. The red-stained positive neurons were measured with image-analysis software (DMBL QWin, Leica) and expressed as percentages. The average percentage of 5 serial slides represented the score of positive neurons in a specimen.

\section{In Situ DNA End-labeling Detection (TUNEL)}

The paraffin-embedded tissue sections $(4 \mu \mathrm{m})$ of parietal cortex and hippocampus (CA1) biopsy specimens were made in the same way. Terminal deoxynucleotidyl transferase-mediated deoxyuridine triphosphate nick end-labeling (TUNEL) is a staining method used to assist in the identification of apoptotic cells in tissue sections. ${ }^{19}$ The cells showing karyorrhexis and TUNEL positivity in TUNEL-staining sections are markers of apoptosis. ${ }^{20}$ The sections were pretreated as described by Labat-Moleur and coworkers $^{19}$ for the enhanced sensitivity. A Boehringer Mannheim kit (In Situ Cell Death Detection Kit, Peroxidase) was used according to the manufacturer's instructions. The brown-stained positive neurons were measured with image-analysis software (DMBL QWin, Leica) and expressed as percentages. The average percentage of 5 serial slides represented the score of positive neurons in a specimen.

\section{Western Blot Analysis of PARP-1}

After 2 hours of weaning, the rabbits were killed. The brains were excised, and parietal cortex and hippocampus CA1 biopsy specimens were dissected and snap-frozen. The nuclear and cytoplasmic proteins of the parietal cortex and hippocampus were extracted as described by Toliver-Kinsky and colleagues. ${ }^{21}$ Fifty micrograms of protein was electrophoresed through a $7.5 \%$ sodium dodecylsulfate-polyacrylamide gel. The proteins were transferred to polyvinylidene fluoride membranes (Amersham Biosciences) overnight. The membranes were washed and blocked in a PBS solution containing $10 \%$ nonfat dry milk, $1 \%$ normal goat serum, $0.1 \%$
Tween-20, and $0.0001 \%$ thimerosal. The membranes were incubated in the presence of mouse anti-PARP-1 (dilution 1:1000, clone: C-2-10, Zymed Labs) at $4{ }^{\circ} \mathrm{C}$ overnight. The membranes were completely washed with PBS containing $0.1 \%$ Tween-20 before addition of the horseradish peroxidase-conjugated goat anti-mouse secondary antibody (Vector Labs) diluted 1:5000. After a 1- to 1.5-hour incubation in the secondary antibody solution, the membranes were washed for 7 to 5 minutes with PBS. The protein was visualized with the ECL Plus Western blot detection system, according to the manufacturer's instructions (Amersham Biosciences). Cleaved PARP was identified in some samples but was not quantified in this study. Only nuclear extracts were analyzed for PARP-1.

\section{Statistical Analysis}

The mean from the microdialysis samples of each group in a conscious state was used as a baseline value. The levels of microdialysis samples, corrected for in vitro recovery of the probe, are expressed as multiples of basal values. All data are presented as means \pm standard error of the mean (SEM) or means \pm standard deviation. Different groups were compared by using the Student $t$ test for unpaired data. A repeated-measures analysis of variance with the Wilcoxon signed-rank test was used for comparison between relevant time points and baseline values in the same group. Statistical analyses were carried out with SPSS 10.0 software (SPSS, Inc).

\section{Results}

Changes of Ratios of Lactate/Glucose, Lactate/Pyruvate, and Extracellular Glutamate Concentration in the Hippocampal Domain

All values are presented as mean multiples of basal levels. Mean basal levels of glucose $( \pm$ SEM) were as follows: $3.92 \pm 0.61 \mathrm{mmol} / \mathrm{L}$ in the DHCA group $(\mathrm{n}=15)$ and 3.88 $\pm 0.88 \mathrm{mmol} / \mathrm{L}$ in the $\mathrm{CPB}$ group $(\mathrm{n}=15 ; P>.05)$. Those of lactate $( \pm$ SEM) were as follows: $7.02 \pm 2.3 \mathrm{mmol} / \mathrm{L}$ in the DHCA group and $6.92 \pm 2.8 \mathrm{mmol} / \mathrm{L}$ in the $\mathrm{CPB}$ group $(P>.05)$. Those of pyruvate $( \pm$ SEM) were as follows: $348.21 \pm 25.43 \mu \mathrm{mol} / \mathrm{L}$ in the DHCA group and $331.02 \pm$ $30.28 \mu \mathrm{mol} / \mathrm{L}$ in the $\mathrm{CPB}$ group $(P>.05)$. Those of glutamate $( \pm$ SEM) were as follows: $17.02 \pm 6.3 \mu \mathrm{mol} / \mathrm{L}$ in the DHCA group and $17.22 \pm 6.8 \mu \mathrm{mol} / \mathrm{L}$ in the CPB group $(P>.05)$.

There was a significant increase in the ratio of lactate/ pyruvate and lactate/glucose from the beginning of weaning in the DHCA group when compared with values in the CPB group and baseline values $(P<.05$, Figures E1 and E2). The top values of lactate/pyruvate and lactate/glucose ratios in the DHCA group were $5.01 \pm 0.6$ and $4.32 \pm 0.7$ times the basal level, respectively $(P<.05)$. The 2 ratios were decreasing from maximal values, but during observation, the values of the DHCA group were still significantly higher than those of the CPB group and its basal level $(P<.05)$.

The effect of DHCA on the extracellular level of glutamate in the DHCA group was significant. As shown in Figure E3, there was a significant increase in the extracel- 
lular level of glutamate from the beginning of rewarming when compared with values in the CPB group and the baseline value $(P<.05)$. The top values in the DHCA groups were up to $8.43 \pm 1.3$ times the basal level. The value of glutamate in the CPB group was stable during the whole observational time, but after weaning of $\mathrm{CPB}$, the values of the DHCA group decreased relative to baseline levels.

\section{Histopathologic Findings}

More damaged neurons were observed in the DHCA group than in CPB group (Figure E4). The total histopathologic scores of the DHCA and CPB groups were $33.6 \pm 4.2$ and $15.4 \pm 2.1$ (mean \pm standard deviation), respectively, with significant differences $(P=.005)$. The injured cells in the hippocampus CA1 and parietal cortex of the DHCA group presented primarily with edema, swollen pyramidal neurons with highly eosinophilic cytoplasm, and few shrunken neurons with pyknotic nuclei, which were indicative of cell necrosis.

\section{PARP Activity}

PARP activity could be reflected by the positive extent of PAR immunostaining. There was weak PAR immunostaining at the parietal cortex and hippocampus (CA1) regions in the CPB group. Extensive and strongly positive PAR immunostaining was observed in the DHCA group (Figure E5). The ratio of PAR-positive staining after DHCA was more than that seen in the CPB group $(41.2 \% \pm 4.5 \%$ vs $16.7 \% \pm 3.4 \%$ in the cortex, $P<.05 ; 54.3 \% \pm 6.2 \%$ vs $18.8 \% \pm 4.2 \%$ in the hippocampus; $P<.05$ ).

\section{Detection of Apoptotic Cells (TUNEL Staining)}

TUNEL-positive cells demonstrated 1 or more brownstained substrates, which might be broken DNA fragments, chromatin margination, or nuclear condensation in neurons. TUNEL staining is too weak to completely identify apoptosis from necrosis. The positive staining only showed the high probability of apoptosis. ${ }^{20}$ TUNEL-positive cells were identified at the parietal cortex and hippocampus (CA1) regions (Figure E6). The ratios of apoptotic cells increased after DHCA versus CPB $(3.0 \% \pm 0.2 \%$ vs $0.4 \% \pm 0.08 \%$ in the cortex $[P<0.05]$ and $1.3 \% \pm 0.3 \%$ vs $0.1 \% \pm$ $0.03 \%$ in the hippocampus $[P<.05]$, respectively.

\section{Western Blot Analysis (PARP-1)}

Another characteristic that is a sensitive marker of the onset of apoptosis is the cleavage of PARP from a 116-kd polypeptide to an $89-\mathrm{kd}$ fragment in cells. ${ }^{22}$ No PARP cleavage was observed in any of the CPB group, but there was PARP cleavage to an 89-kd fragment at the parietal cortex and hippocampus (CA1) regions in the DHCA group (Figure E7).

\section{Discussion}

To date, because of the uncertain pathogenesis about neuronal injury after DHCA, neurologic morbidity associated with DHCA remains high, afflicting 5\% to $40 \%$ of postoperative survivors. ${ }^{1-3}$ Under such circumstances, we explored the potential mechanism of neuronal injury after DHCA by setting up a model of DHCA linked with cerebral microdialysis. Because the cerebrovascular anatomy and many parameters of rabbits are nearly similar to those of human subjects, ${ }^{23,24}$ we chose the rabbit as our animal model. Furthermore, the maneuver of setting up CPB and the lower ratio of priming volume to whole blood volume in the model were very similar to the clinical setting. Therefore, this model was applicable to studies of brain protection associated with DHCA.

By linking with the model of cerebral microdialysis, we could continuously monitor the concentration changes of glucose, lactate, pyruvate, and glutamate in the interstitial space of intact tissue in vivo. ${ }^{25}$ The lactate/pyruvate and lactate/glucose ratios are excellent markers of ischemia and energy failure in the brain. ${ }^{26}$ Glutamate is one of the major excitatory neurotransmitters in the central nervous system. Glutamate overrelease would lead to excitotoxicity. ${ }^{4}$ In this study cerebral microdialysis was associated with the model, which afforded an effective way to study brain molecular changes. The lactate/pyruvate and lactate/glucose ratios were significantly increased after reperfusion in the DHCA group compared with those of the CPB group, which was indicative of ischemia and energy metabolic disorder. It can be seen from Figure E3 that the increasing extent of glutamate was significant and of a high magnification in a short time, which could induce the excitotoxicity in the DHCA group.

We found that PARP-1 was overactivated in the DHCA group, which was indicated by the strongly positive expression of PAR. A worthwhile point is that overactivation of PARP-1 after DHCA was not investigated in previous animal models or clinical research. In addition, more neuronal injury, including necrosis and apoptosis, was demonstrated in the DHCA group compared with the CPB group by means of morphologic studies, TUNEL staining, and Western blot analysis for cleavage of PARP-1. Therefore, the experimental results demonstrated that there were molecular events in neuronal injury, which included simultaneous cellular energy failure, excitotoxicity, overactivation of PARP-1, and neuronal necrosis and/or apoptosis.

Several molecular mechanisms were responsible for the pathogenesis of ischemic neuronal injury, but most data available were obtained from normothermic brain ischemia models. Now the following mechanism is becoming a wellaccepted pathogenesis in ischemic brain injury. ${ }^{13-16}$ In the ischemic brain energy deprivation triggers glutamate release and overactivation of neuronal glutamate receptors, which 
results in increases of intracellular calcium and activation of calcium-dependent enzymes, followed by overproduction of nitric oxide synthase and other reactive oxygen species. These reactive oxygen species ultimately damage cellular DNA and result in excitotoxicity. Massive DNA damage can also directly activate nuclear PARP-1 activity, which transforms $\mathrm{NAD}^{+}$into long polymers of PAR and nicotinamide and causes drastic depletion of the pyridine nucleotide pool. Under these stressful conditions, the NAD salvage pathway is activated with resynthesis of $\mathrm{NAD}^{+}$. Because the synthesis procedure uses much ATP, DNA damagedependent PARP-1 overactivation ultimately depletes cellular ATP pools. These events cause a vicious cycle, leading to aggravated energy failure and neuronal death. For a clear vision of the cascade, a molecular pathway to the neuronal injury after DHCA was described as follows: cellular energy failure $\rightarrow$ excitotoxicity $\rightarrow$ DNA damage $\rightarrow$ overactivation of PARP-1 $\rightarrow$ necrosis and apoptosis. ${ }^{13,16}$ In this study, we proved that some key molecular mediators, such as cellular energy deficit, the overrelease of glutamate, overactivation of PARP-1, and neuronal necrosis and/or apoptosis, also existed in neuronal injury after DHCA in a rabbit model. This implied that there could be a similar molecular pathway in neuronal injury after DHCA.

In recent years, overactivation of the DNA damage-dependent nuclear enzyme PARP-1 has become a central event in ischemia-induced neurodegeneration. Overactivation of PARP-1 has been known as a downstream event of excitotoxicity, which is more approximate to cell damage or death. ${ }^{27}$ Given the overactivation of PARP-1 to the pathogenesis in ischemic brain injury, a series of strategies, such as gene deletion and chemical inhibitors, have been used to suppress the PARP-1 activity. Many researches have proved the same result that remarkable neuroprotection is obtained by suppression of poly(ADP-ribosyl)ation in the ischemic brain. A pioneering study showed that gene knockout of PARP provided massive neuroprotection against glutamate-NO-mediated ischemic insults and obviously reduced infarct volume (up to 80\%) induced by middle cerebral artery occlusion. ${ }^{28}$ Another study reported that the infarct volume caused by ischemia-reperfusion injury is significantly smaller $45 \%$ in PARP-1 knockout mice than that in wild-type mice. ${ }^{29}$ In recent years, many potent water-soluble PARP inhibitors, such as $\mathrm{N}$-(6-oxo-5,6dihydro-phenanthridin-2-yl)-N,N-dimethylacetamide (PJ-34), indeno-isoquinolinone (INO-1001), and 4-(4-(N,N-dimethylaminomethyl)phenyl)-5-hydroxy isoquinolinone (KCL-440), demonstrated neuroprotective effect in models of brain ischemia. ${ }^{30,31}$ In light of the abovementioned studies, we are delighted to find that overactivation of PARP-1 followed DHCA in this study. To suppress the poly(ADP-ribosyl)ation would be one of the most promising strategies against neuronal injury after DHCA to date. We intend to study the protective effect of PARP-1 inhibitor on neuronal injury after DHCA in the future.
However, in this study we investigated only the wellknown molecular events in neuronal injury after DHCA, which were still not composed of a complete pathway. Further research needs to be done to explain the causal relationship between the events. Because the study used a rabbit model, which is not an ideal subject, it would be a stretch to extrapolate the findings to subsequent clinical guidelines.

In summary, we have demonstrated that the process of DHCA results in an overactivation of PARP. We have also found that molecular events existed simultaneously in the same model (ie, cellular energy failure, overrelease of glutamte, overactivation of PARP-1, and necrosis and/or apoptosis). Furthermore, this study offers the expectation that PARP-1 inhibitors might play a neuroprotective role in DHCA.

\section{References}

1. Bellinger DC, Jonas RA, Rappaport LA, Wypij D, Wernovsky G, Kuban KC, et al. Developmental and neurologic status of children after heart surgery with hypothermic circulatory arrest or low-flow cardiopulmonary bypass. $N$ Engl J Med. 1995;332:549-55.

2. Wypij D, Newburger JW, Rappaport LA, duPlessis AJ, Jonas RA, Wernovsky G, et al. The effect of duration of deep hypothermic circulatory arrest in infant heart surgery on late neurodevelopment: the Boston Circulatory Arrest Trial. J Thorac Cardiovasc Surg. 2003;126: 1397-403.

3. Gaynor JW, Nicolson SC, Jarvik GP, Wernovsky G, Montenegro LM, Burnham NB, et al. Increasing duration of deep hypothermic circulatory arrest is associated with an increased incidence of postoperative electroencephalographic seizures. J Thorac Cardiovasc Surg. 2005; 130:1278-86

4. Hugon J, Vallat JM, Dumas M. [Role of glutamate and excitotoxicity in neurologic diseases]. Rev Neurol (Paris). 1996;152:239-48.

5. Amir G, Ramamoorthy C, Riemer RK, Reddy VM, Hanley FL. Neonatal brain protection and deep hypothermic circulatory arrest: pathophysiology of ischemic neuronal injury and protective strategies. Ann Thorac Surg. 2005;80:1955-64.

6. Johnston MV, Trescher WH, Ishida A, Nakajima W. Neurobiology of hypoxic-ischemic injury in the developing brain. Pediatr Res. 2001; 49:735-41.

7. Choi DW, Rothman SM. The role of glutamate neurotoxicity in hypoxic-ischemic neuronal death. Annu Rev Neurosci. 1990;13:17182.

8. Tseng EE, Brock MV, Kwon CC, Annanata M, Lange MS, Troncoso $\mathrm{JC}$, et al. Increased intracerebral excitatory amino acids and nitric oxide after hypothermic circulatory arrest. Ann Thorac Surg. 1999;67: 371-6.

9. Leist M, Nicotera P. Apoptosis, excitotoxicity, and neuropathology. Exp Cell Res. 1998;239:183-201.

10. Kurth CD, Priestley M, Golden J, McCann J, Raghupathi R. Regional patterns of neuronal death after deep hypothermic circulatory arrest in newborn pigs. J Thorac Cardiovasc Surg. 1999;118:1068-77.

11. Ditsworth D, Priestley MA, Loepke AW, Ramamoorthy C, McCann J, Staple L, et al. Apoptotic neuronal death following deep hypothermic circulatory arrest in piglets. Anesthesiology. 2003;98:1119-27.

12. Lipton SA, Rosenberg PA. Excitatory amino acids as a final common pathway for neurologic disorders. N Engl J Med. 1994;330:613-22.

13. Chiarugi A. Poly(ADP-ribosyl)ation and stroke. Pharmacol Res. 2005; 52:15-24.

14. D'Amours D, Desnoyers S, D'Silva I, Poirier GG. Poly(ADP-ribosy1)ation reactions in the regulation of nuclear functions. Biochem $J$. 1999;342(suppl):249-68. 
15. Pieper AA, Verma A, Zhang J, Snyder SH. Poly (ADP-ribose) polymerase, nitric oxide and cell death. Trends Pharmacol Sci. 1999;20: 171-81.

16. Virag L, Szabo C. The therapeutic potential of poly(ADP-ribose) polymerase inhibitors. Pharmacol Rev. 2002;54:375-429.

17. Sawyer CH, Everett JW, Green JD. The rabbit diencephalon in stereotaxic coordinates. J Comp Neurol. 1954;101:801-24.

18. Szabo G, Bahrle S, Stumpf N, Sonnenberg K, Szabo EE, Pacher P, et al. Poly(ADP-ribose) polymerase inhibition reduces reperfusion injury after heart transplantation. Circ Res. 2002;90:100-6.

19. Labat-Moleur F, Guillermet C, Lorimier P, Robert C, Lantuejoul S, Brambilla E, et al. TUNEL apoptotic cell detection in tissue sections: critical evaluation and improvement. J Histochem Cytochem. 1998;46:327-34.

20. Takizawa Y, Takashima S, Itoh M. A histopathological study of premature and mature infants with pontosubicular neuron necrosis: neuronal cell death in perinatal brain damage. Brain Res. 2006;1095:200-6.

21. Toliver-Kinsky T, Papaconstantinou J, Perez-Polo JR. Age-associated alterations in hippocampal and basal forebrain nuclear factor kappa B activity. J Neurosci Res. 1997;48:580-7.

22. Graziani G, Szabo C. Clinical perspectives of PARP inhibitors. Pharmacol Res. 2005;52:109-18.

23. Scremin OU, Sonnenschein RR, Rubinstein EH. Cerebrovascular anatomy and blood flow measurements in the rabbit. J Cereb Blood Flow Metab. 1982;2:55-66.
24. Orr JA, DeSoignie RC, Wagerle LC, Fraser DB. Regional cerebral blood flow during hypercapnia in the anesthetized rabbit. Stroke. 1983; $14: 802-7$.

25. Plock N, Kloft C. Microdialysis-theoretical background and recent implementation in applied life-sciences. Eur J Pharm Sci. 2005;25:1-24.

26. Goodman JC, Valadka AB, Gopinath SP, Uzura M, Robertson CS. Extracellular lactate and glucose alterations in the brain after head injury measured by microdialysis. Crit Care Med. 1999;27:196573.

27. Koh DW, Dawson TM, Dawson VL. Mediation of cell death by poly(ADP-ribose) polymerase-1. Pharmacol Res. 2005;52:5-14.

28. Eliasson MJ, Sampei K, Mandir AS, Hurn PD, Traystman RJ, Bao J, et al. Poly(ADP-ribose) polymerase gene disruption renders mice resistant to cerebral ischemia. Nat Med. 1997;3:1089-95.

29. Endres M, Wang ZQ, Namura S, Waeber C, Moskowitz MA. Ischemic brain injury is mediated by the activation of poly(ADP-ribose)polymerase. J Cereb Blood Flow Metab. 1997;17:1143-51.

30. Besson VC, Zsengeller Z, Plotkine M, Szabo C, Marchand-Verrecchia C. Beneficial effects of PJ34 and INO-1001, two novel water-soluble poly(ADP-ribose) polymerase inhibitors, on the consequences of traumatic brain injury in rat. Brain Res. 2005;1041:149-56.

31. Ikeda Y, Hokamura K, Kawai T, Ishiyama J, Ishikawa K, Anraku T, et al. Neuroprotective effects of KCL-440, a new poly(ADP-ribose) polymerase inhibitor, in the rat middle cerebral artery occlusion model. Brain Res. 2005;1060:73-80. 


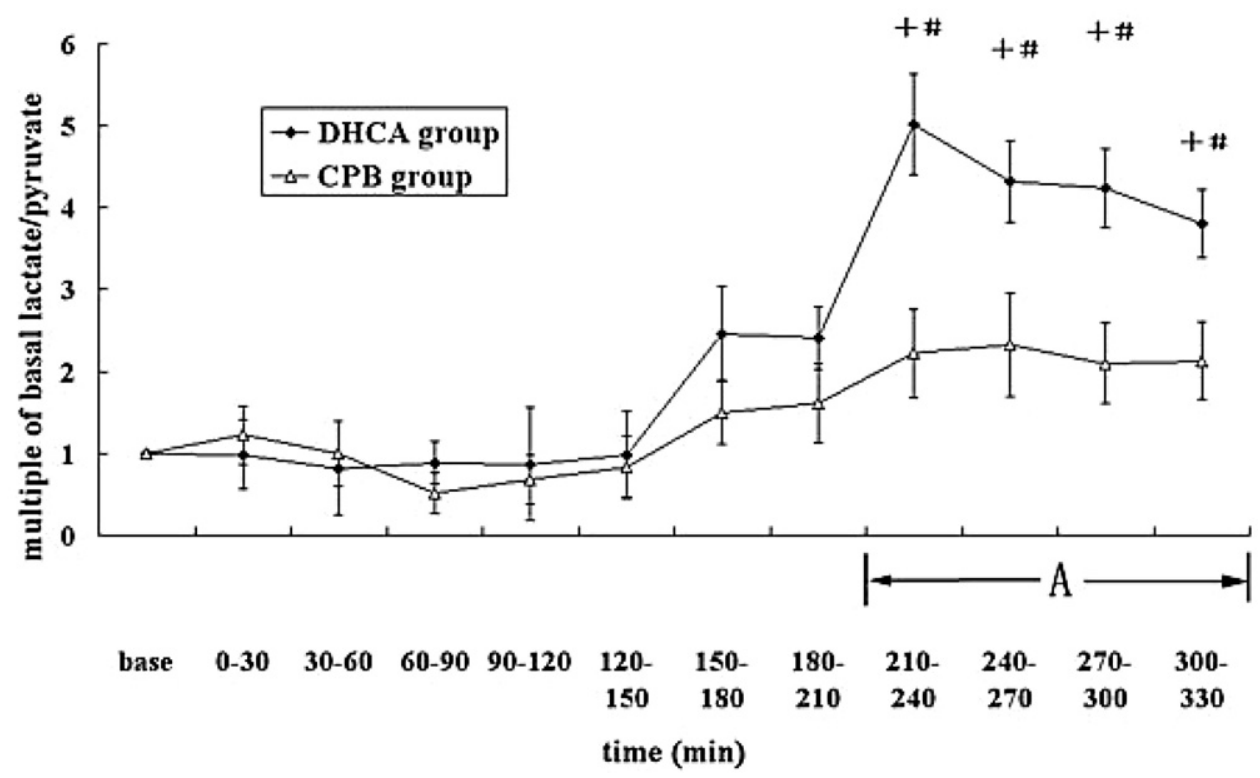

Figure E1. Time course of dialysate lactate/pyruvate ratio. DHCA, Deep hypothermic circulatory arrest; $C P B$, cardiopulmonary bypass; $A$, recovery from CPB. $+P<.05$, difference between relevant time points and baseline in the same group. \#P<.05, difference between the CPB and DHCA groups.

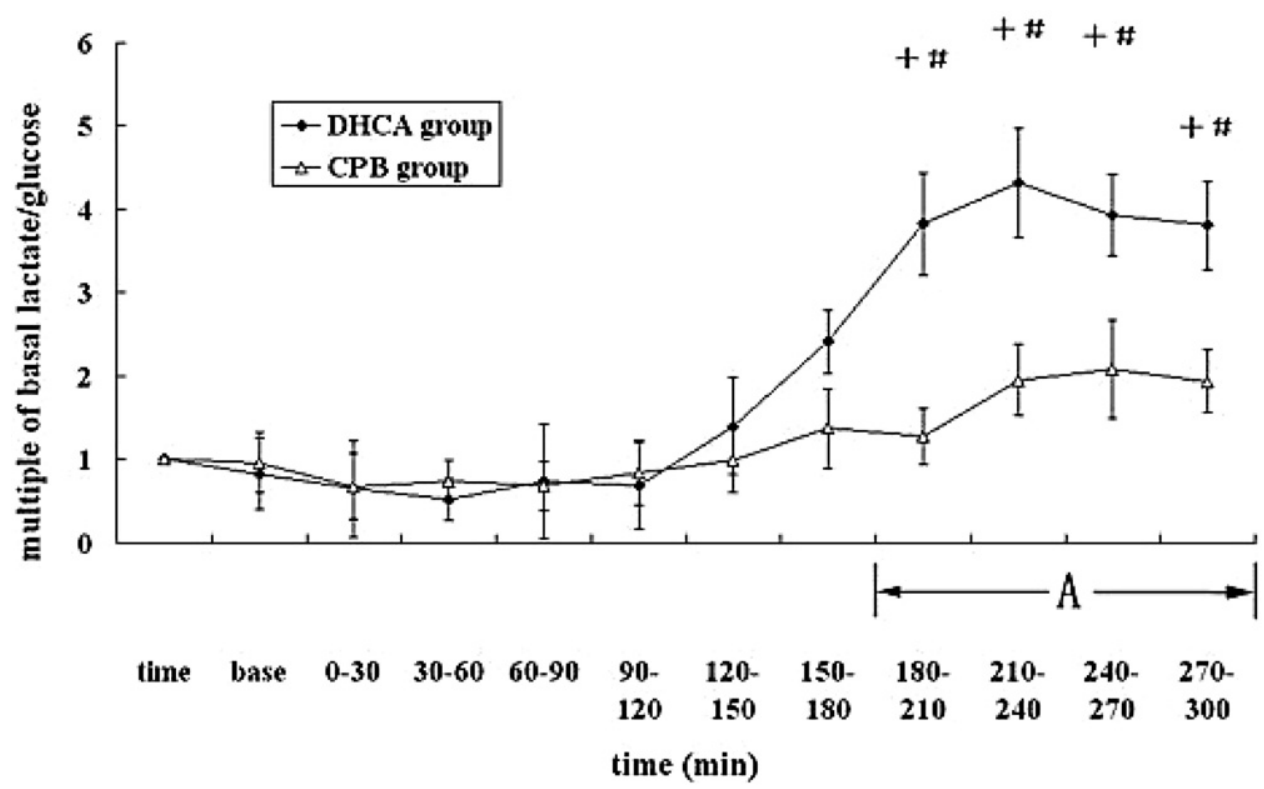

Figure E2. Time course of dialysate lactate/glucose ratio. DHCA, Deep hypothermic circulatory arrest; $C P B$, cardiopulmonary bypass; $A$, recovery from CPB. $+P<.05$, difference between relevant time points and baseline in the same group. \#P<.05, difference between the CPB and DHCA groups. 


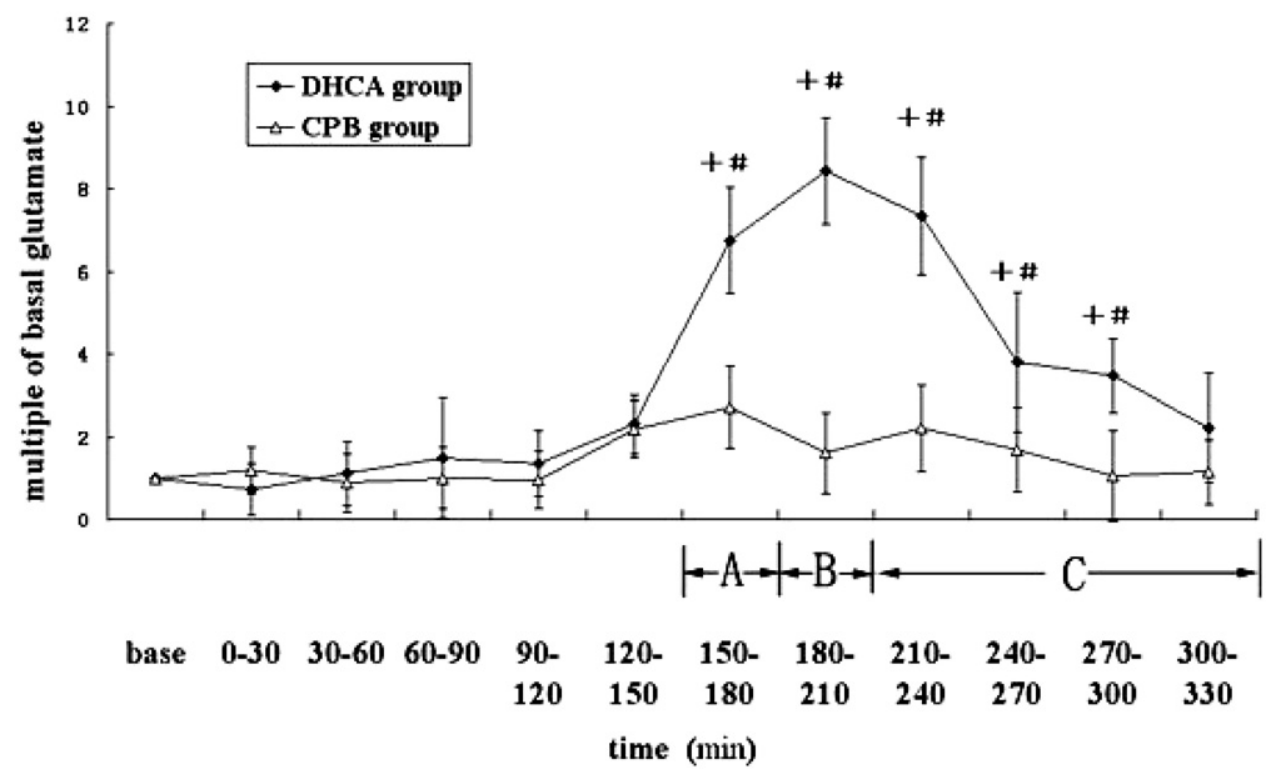

Figure E3. Time course of dialysate glutamate level. DHCA, Deep hypothermic circulatory arrest; $C P B$, cardiopulmonary bypass; $A$, rewarming; $B$, assisted circulation; $C$, recovery from $C P B$. $+P<.05$, difference between relevant time points and baseline in the same group. $\# P<.05$, difference between the CPB and DHCA groups. 


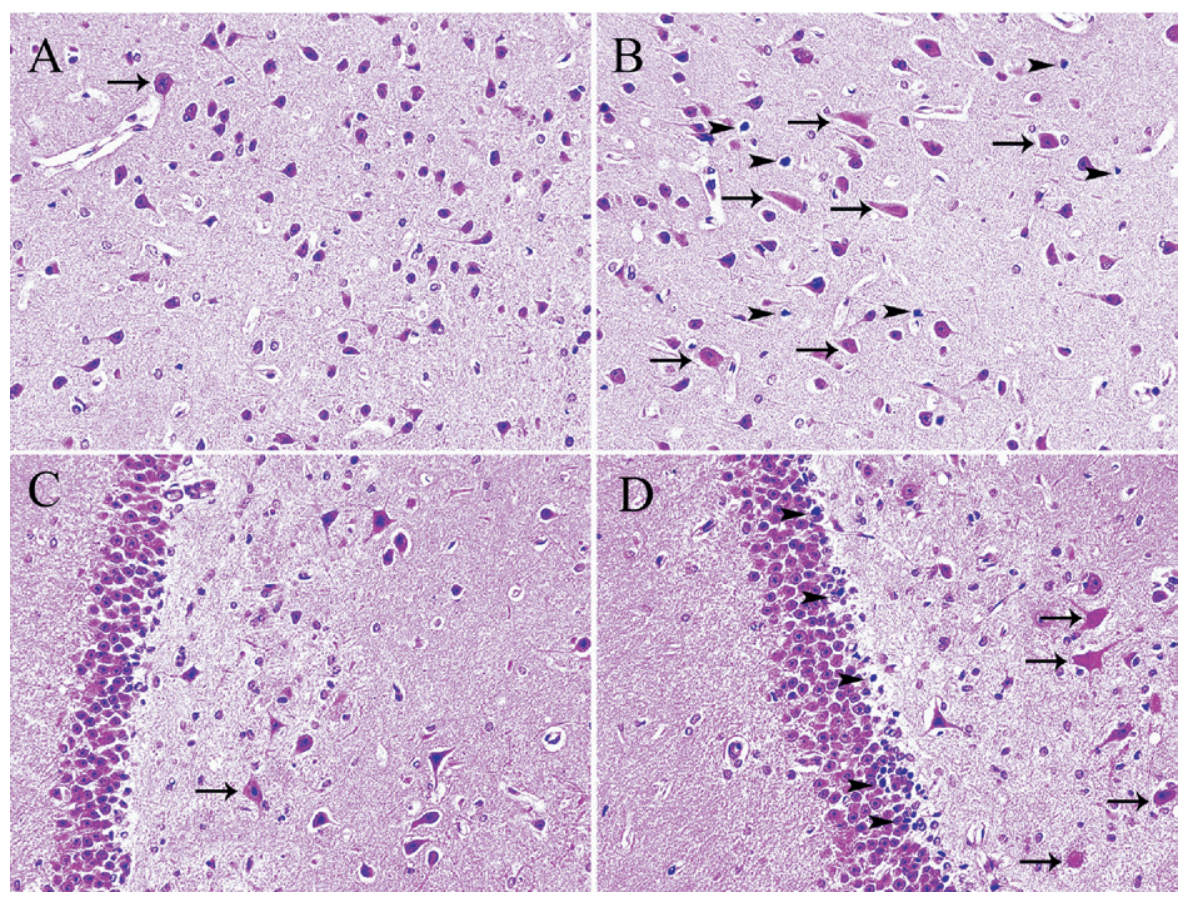

Figure E4. Histopathologic findings of parietal cortex (A and B) and hippocampus CA1 (C and D) specimens stained with hematoxylin and eosin. (Original magnification $400 \times$.) The arrows point to swollen cells with highly eosinophilic cytoplasm. The arrowheads point to shrunken cells with pyknotic nuclei. The damaged neurons with marked eosinophilic cytoplasm and pyknotic nuclei in the deep hypothermic circulatory arrest group (B and D) were more than in the cardiopulmonary bypass group ( $A$ and $C$ ). 


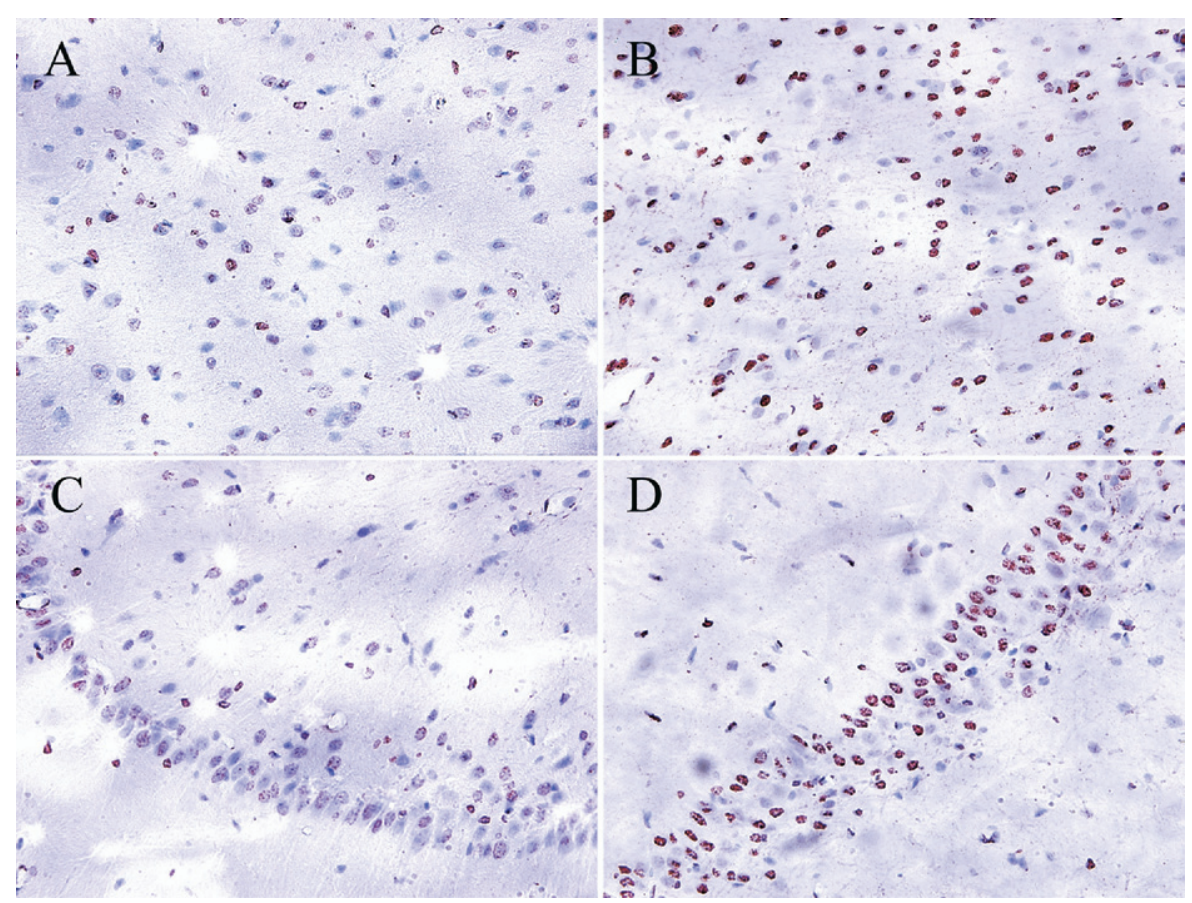

Figure E5. Immunohistological staining against poly(adenosine diphosphate-ribose). Positive immunostaining of poly(adenosine diphosphate-ribose) in the parietal cortex ( $A$ and $B$ ) and hippocampus CA1 (C and D) specimens demonstrated red color. (Original magnification $400 \times$.) Poly(adenosine diphosphate-ribose) formation was obviously increased in the deep hypothermic circulatory arrest group (B and D) compared with the cardiopulmonary bypass group ( $A$ and $C)$. 


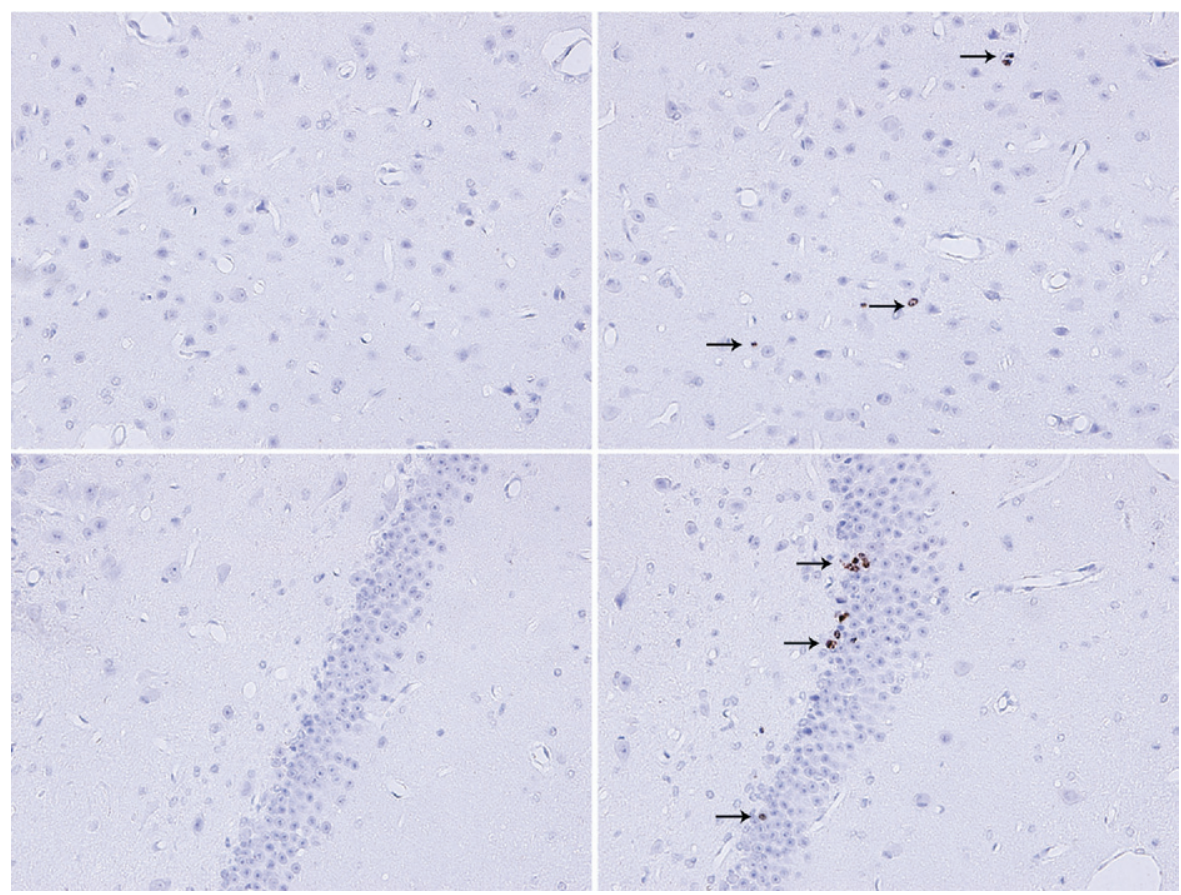

Figure E6. Terminal deoxynucleotidyl transferase-mediated deoxyuridine triphosphate nick end-labeling staining. Positive immunostaining neurons in the parietal cortex (A and B) and hippocampus CA1 (C and D) specimens demonstrated brown color. (Original magnification $400 \times$.) A few stained neurons were shown in the deep hypothermic circulatory arrest group ( $B$ and $D$ ) but not in the cardiopulmonary bypass group ( $A$ and $C$ ). The arrows point to terminal deoxynucleotidyl transferase-mediated deoxyuridine triphosphate nick end-labeling-positive staining, with brown color indicative of apoptosis.

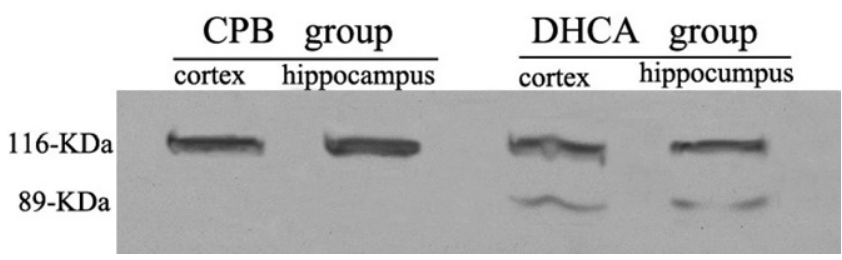

Figure E7. Western blot analysis of poly(adenosine phosphate-ribose) polymerase 1 in the parietal cortex and hippocampus CA1. The deep hypothermic circulatory arrest (DHCA) lane demonstrated 2 bands of full-length poly(adenosine phosphate-ribose) polymerase 1 (116 Kd) and cleaved $(89 \mathrm{Kd})$, but the cardiopulmonary bypass (CPB) lane showed only 1 poly(adenosine phosphate-ribose) polymerase 1 band (116 Kd). 